\title{
Gastrectomía vertical versus by-pass gástrico en Y de Roux: resultados a corto y medio plazo
}

\author{
Laura Marti-Gelonch ${ }^{1}$, José Ignacio Asensio-Gallego ${ }^{1}$, Santiago Larburu-Echániz ${ }^{1}$, \\ Emma Eizaguirre-Letamendia ${ }^{1}$ y José María Enríquez-Navascués ${ }^{1}$
}

\begin{abstract}
Sleeve gastrectomy vs gastric bypass: results in the short and medium term
Introduction: Sleeve gastrectomy (SG) and gastric bypass (GBP) are the most commonly used procedures in the treatment of obesity. The objective of this paper is to compare these two techniques in regard to weight loss and improvement of the associated comorbidities. Material and Method: An ambispective observational study of patients undergoing GBP and SG from January 2011 to December 2013. Weight loss, BMI, success rate, weight regain, impact on associated comorbidities and morbimortality of both techniques were analysed. Results: 172 patients underwent operations (92 GBP and 80 SG). GBP had better results in regard to weight loss and improvement of comorbidities. The SG group had greater weight regain. The two procedures had similar results in regard to morbimortality.
\end{abstract}

Key words: obesity; comorbidities; surgery.

\section{Resumen}

Introducción: La gastrectomía vertical (GV) y el by-pass gástrico (BPG) son los procedimientos más utilizados en el tratamiento de la obesidad. El objetivo de este trabajo es comparar ambas técnicas tanto en pérdida ponderal como en mejoría de las comorbilidades asociadas. Materiales y Método: Estudio observacional ambispectivo de los pacientes sometidos a BPG y GV desde enero de 2011 hasta diciembre de 2013. Se ha analizado la pérdida de peso y de IMC, la tasa de éxito, la reganancia de peso, el impacto en las comorbilidades asociadas y la morbimortalidad de ambas técnicas. Resultados: 172 pacientes fueron intervenidos (92 BPG y $80 \mathrm{GV}$ ). El BPG presenta mejores resultados en cuanto a pérdida de peso y mejoría de las comorbilidades. El grupo GV presenta mayor reganancia de peso. Ambos procedimientos presentan resultados similares en cuanto a morbimortalidad.

Palabras clave: obesidad; comorbilidades; cirugía.
Hospital Universitario Donostia. San Sebastián, España.

Conflictos de interés: no hay. Recibido el 15 de marzo de 2018 y aceptado para publicación el 28 de mayo de 2018.

Correspondencia a: Dra. Laura Marti Gelonch lauramartig_@hotmail.com

\section{Introducción}

La obesidad y el sobrepeso son problemas crecientes en todo el mundo en las últimas décadas, afectando a un $36,9 \%$ de los hombres y a un $38 \%$ de las mujeres en el mundo occidental ${ }^{1,2}$. La obesidad es una enfermedad crónica, que asocia una mayor mortalidad y morbilidad derivada de comorbilidades como la diabetes mellitus tipo II (DM-II), la hipertensión arterial (HTA), la dislipemia, el aumento del riesgo cardiovascular, síndrome de apnea obstructiva del sueño (SAOS), cáncer y otros.

La pérdida de peso se asocia a una mejoría o resolución de las comorbilidades y a una reducción de la mortalidad. Numerosos tratamientos han sido de- sarrollados para hacer frente a la obesidad mórbida, como medidas diéteticas y conductuales, fármacos y procedimientos endoscópicos y quirúrgicos.

Diversos estudios revelan que el tratamiento quirúrgico aporta una mayor pérdida de peso y una mayor tasa de remisión de comorbilidades que el tratamiento dietético y farmacológico ${ }^{3}$. Entre los procedimientos quirúrgicos descritos, el by-pass gástrico $(\mathrm{BPG})$ y la gastrectomía vertical $(\mathrm{GV})$ son los más empleados. A nivel mundial, la GV es el procedimiento más empleado superando al BPG, técnica más empleada hasta $2015^{4}$.

Estudios publicados han demostrado buenos resultados con el empleo de GV en cuanto a pérdida de peso y resolución de comorbilidades, siendo un 
procedimiento menos exigente técnicamente y con una menor tasa de complicaciones ${ }^{5,6}$.

Por otro lado, la reganancia de peso es una de las complicaciones asociada a la cirugía bariátrica y de origen multifactorial, siendo reportadas tasas muy heterogéneas al respecto ${ }^{7,8}$.

El objetivo de este trabajo es comparar los resultados clínicos entre BPG y GV en términos de porcentaje de IMC perdido (PIMCP) y porcentaje de exceso de IMC perdido (PEIMCP), tasa de reganancia de peso con ambas técnicas quirúrgicas, morbimortalidad asociada a ambos procedimientos y mejoría o resolución de las comorbilidades asociadas a la obesidad.

\section{Materiales y Método}

Estudio ambispectivo unicéntrico sobre una base de datos prospectiva de la Sección de Cirugía Esófago-Gástrica, de los pacientes sometidos a cirugía bariátrica en un periodo que comprende desde enero de 2011 hasta diciembre de 2013. El estudio ha sido aprobado por el comité de ética de nuestro centro.

\section{Evaluación perioperatoria}

Los pacientes sometidos a cirugía bariátrica durante el período de estudio, fueron evaluados por un comité multidisciplinar tanto pre como posoperatoriamente, formado por endocrinólogos, psiquiatras, anestesiólogos, enfermería y cirujanos.

Se han realizado dos tipos de técnicas, la GV y el $\mathrm{BPG}$, indicando la primera, para pacientes con IMC de 40-45, no picoteadores y como primera fase en pacientes superobesos con IMC $\geq 55$, mientras que los pacientes con un IMC de 40-45, sin grandes comorbilidades y catalogados como picoteadores, eran sometidos a BPG.

Se realiza un seguimiento medio de 24-36 meses (12-48 meses). Se evalúan, por una parte, los resultados de las curvas ponderales en forma de PIMCP y PEIMCP de estos pacientes. Por otra parte, se analizan las tasas de éxito y fracaso con ambas técnicas, la morbimortalidad asociada a ambos procedimientos (mediante la clasificación de Clavien-Dindo) y el impacto de la cirugía en comorbilidades como la HTA, la DM-II y el SAOS.

\section{Técnica quirúrgica}

Todas las intervenciones fueron realizadas por el mismo equipo quirúrgico (formado por tres cirujanos) y mediante abordaje laparoscópico.

En el caso del BPG, se confecciona un reservorio gástrico aislado con un asa biliar en $\mathrm{Y}$ de Roux de
$60 \mathrm{~cm}$ y un asa alimentaria de $150 \mathrm{~cm}$ antecólica. $\mathrm{La}$ anastomosis gastroyeyunal se realiza con endograpadora circular de $25 \mathrm{~mm}$ y la anastomosis yeyunoyeyunal latero-lateral mecánica.

En la $\mathrm{GV}$, se realiza una resección gástrica vertical sobre una sonda tutor de 36 French desde 4-5 cm del píloro hasta el ángulo de Hiss, mediante endograpadora.

En ambos procedimientos se realiza un test de fugas mediante la administración de azul de metileno a través de una sonda nasogástrica y se deja un drenaje aspirativo intraabdominal.

\section{Cuidados perioperatorios}

Todos los pacientes reciben profilaxis antitrombótica con heparina de bajo peso molecular y medias de compresión neumática durante el procedimiento, además de profilaxis antibiótica con amoxicilinaclavulánico $2 \mathrm{~g}$ (en caso de alergia, clindamicina $900 \mathrm{mg}+$ gentamicina $240 \mathrm{mg}$ ).

En los BPG, se inicia movilización el primer día posoperatorio (PO). Se realiza un estudio baritado de control a las 48 horas y en caso de no objetivar fuga de contraste, se retira el drenaje y se inicia dieta líquida al tercer día PO, siendo dado de alta a domicilio al quinto día PO.

En la GV, se inicia movilización y tolerancia a sorbos de agua el primer día PO. Se retira el drenaje aspirativo al segundo día PO, progresando la dieta a líquidos más lácteos y siendo dados de alta entre el tercer y quinto día PO.

Posteriormente, se realiza seguimiento por parte de cirugía, endocrinología y enfermería cada 3 meses hasta los 24 meses de la intervención. Posteriormente el seguimiento es realizado por enfermería y endocrinología.

\section{Éxito o fracaso de la técnica quirúrgica}

Según los criterios actuales de éxito de la cirugía bariátrica, se ha considerado resultado excelente un PEIMCP $>65 \%$, resultado bueno entre $50-65 \%$ y fracaso si PEIMCP $<50 \%$.

Se ha considerado corto plazo, los resultados obtenidos a 1 año de la cirugía y medio plazo los resultados obtenidos a 3 años de la cirugía bariátrica 9 .

\section{Tasa de reganancia de peso}

En la actualidad no existe una definición universal sobre la reganancia de peso en pacientes sometidos a cirugía bariátrica ${ }^{10}$. En este estudio y siendo la definición más empleada en la actualidad, se ha considerado reganancia de peso el aumento mayor a $10 \mathrm{~kg}$ desde el peso más bajo adquirido tras la cirugía bariátrica. Se han comparado las 
tasas de reganancia con ambas técnicas a corto y medio plazo.

\section{Resolución o mejoría de las comorbilidades}

Se han analizado los resultados de la cirugía bariátrica en cuanto a resolución o mejoría de las comorbilidades según los criterios estandarizados publicados en 2015 por la American Society for Metabolic and Bariatric Surgery (ASMNS) ${ }^{11}$.

\section{Análisis estadístico}

Se ha empleado el paquete estadístico STATA versión 14.0. Se han descrito las variables mediante el estadístico más apropiado para la naturaleza y escala de medición de cada una: media y desviación estándar (o mediana y rango intercuartil) para variables cuantitativas y frecuencias absolutas y relativas en porcentaje para variables cualitativas.

Se ha realizado análisis univariante para evaluar la importancia de las variables de estudio en relación con el resultado/s de interés (outcome). Para las variables paramétricas (Mann-Whitney, Kruskal Wallis) si fueran necesarios y para las variables categóricas se han realizado pruebas chi-cuadrado o prueba exacta de Fischer.

\section{Resultados}

Durante el período de estudio comprendido entre enero de 2011 y diciembre de 2013, un total de 172 pacientes fueron sometidos a cirugía bariátrica $(\mathrm{n}=172)$.

92 pacientes $(53,4 \%)$ fueron sometidos a BPG y $80(46,5 \%)$ pacientes fueron sometidos a $\mathrm{GV}$, con un seguimiento medio de 30 meses (12-48 meses). Las características demográficas de ambos grupos se mencionan en la Tabla 1.

La edad media de los pacientes fue de 44,56 años. En ambos grupos, aproximadamente un tercio de los pacientes eran hombres $(32,6 \%$ en el grupo BPG y $27,5 \%$ en el grupo GV). El IMC inicial en el grupo BPG fue de 47,28 mientras que en el grupo sometido a GV fue de 45,65 ( $\mathrm{p}=0,078)$.

Comparando ambos grupos, no se observan diferencias estadísticamente significativas en cuanto a edad, sexo, IMC inicial y riesgo ASA, ni en cuanto a la distribución de las comorbilidades.

La HTA fue la comorbilidad más frecuente en ambos grupos (35,87\% en BPG y $33,75 \%$ en $\mathrm{GV})$, seguida del SAOS $(29,34 \%$ y $28,75 \%$ respectivamente) y la DM tipo I y II $(21,73 \%$ en grupo BPG y $20 \%$ en el grupo de GV).

No hubo mortalidad relacionada con el proceso quirúrgico. En cuanto a morbilidad y de acuerdo con la clasificación de Clavien-Dindo, un total de 22 pacientes $(12,79 \%)$ sufrieron complicaciones menores (Clavien Dindo I y II) y 8 pacientes $(4,65 \%)$ complicaciones mayores (Claven Dindo III y IV) (Tabla 2).

Al comparar ambas técnicas, existe una mayor tasa de complicaciones en el grupo BPG 24 $(26,09 \%)$ que en el grupo GV, $6(7,5 \%)$. Al realizar análisis por subgrupos, de entre los pacientes que presentan complicaciones, no existen diferencias en cuanto al tipo (menor/mayor) de complicación (prueba exacta de Fisher $\mathrm{p}=0,208$ ).

Tabla 1. Datos demográficos y comorbilidades asociadas

\begin{tabular}{|llll|}
\hline Características & BPG $(\mathbf{n = 9 2 )}$ & $\mathbf{G V}(\mathbf{n}=\mathbf{8 0})$ & valor $\mathbf{p}$ \\
Edad media (DS) & $43,08(11,5)$ & $46,30(10,7)$ & $\mathrm{p}=0,061$ (NS) \\
Sexo n (\%) & & & $\mathrm{p}=0,467$ (NS) \\
Hombres & $30(32,61 \%)$ & $22(27,5 \%)$ & \\
Mujeres & $62(67,39 \%)$ & $58(72,5 \%)$ & \\
IMC inicial (DS) & $47,28(4,88)$ & $45,65(7,11)$ & $\mathrm{p}=0,078$ (NS) \\
Riesgo ASA n (\%) & & & $\mathrm{p}=0,44$ (NS) \\
I & 0 & 1 & \\
II & 35 & 37 & \\
III & 53 & 38 & \\
IV & 4 & 4 & $\mathrm{p}=0,552$ (NS) \\
Comorbilidades n (\%) & $33(35,87 \%)$ & $27(33,75 \%)$ & $\mathrm{p}=0,780$ (NS) \\
HTA & $20(21,73 \%)$ & $16(20 \%)$ & \\
DM & $27(29,34 \%)$ & $23(28,75 \%)$ & $\mathrm{p}=0,931$ (NS) \\
SAOS & & & \\
\hline
\end{tabular}

Tabla 2. Complicaciones mayores y menores en el período posoperatorio precoz

\begin{tabular}{|c|c|c|c|c|}
\hline & & BPG & & \\
\hline Clavien-Dindo & $\mathbf{n}$ & Tipo de complicación & $\mathbf{n}$ & Tipo de complicación \\
\hline I & $\begin{array}{r}10 \\
2 \\
1\end{array}$ & $\begin{array}{l}\text { Infección herida asistencia } \\
\text { Îleo paralítico } \\
\text { Hemorragia intraluminal }\end{array}$ & 1 & Íleo paralítico \\
\hline II & $\begin{array}{l}2 \\
2 \\
1\end{array}$ & $\begin{array}{l}\text { Hemorragia intraluminal } \\
\text { Fuga anastomótica subclínica } \\
\text { Neumonía }\end{array}$ & $\begin{array}{l}1 \\
2\end{array}$ & $\begin{array}{l}\text { ACFA } \\
\text { Hemoperitoneo }\end{array}$ \\
\hline IIIa & 1 & Fuga anastomótica & & \\
\hline IIIb & $\begin{array}{l}2 \\
1 \\
1\end{array}$ & $\begin{array}{l}\text { Hemoperitoneo } \\
\text { Perforación intestinal } \\
\text { Absceso intraabdominal }\end{array}$ & 1 & Perforación intestinal \\
\hline IVa & & & 1 & Distrés respiratorio \\
\hline $\mathrm{IVb}$ & 1 & $\begin{array}{l}\text { Fuga anastomótica } \\
\text { (fallo multiorgánico) }\end{array}$ & & \\
\hline V & 0 & Indeterminante & 0 & Indeterminante \\
\hline
\end{tabular}


Tabla 3. Éxito a corto y medio plazo de la cirugía bariátrica según la técnica empleada

\begin{tabular}{|lcccccc|}
\hline & \multicolumn{3}{c}{ A corto plazo (1 año) } & \multicolumn{3}{c|}{ A medio plazo (3 años) } \\
Resultados & BPG (n= 92) & GT (n= 80) & p & BPG (n= 67) & GT (n= 65) & p \\
Excelente (PEIMCP $>65 \%)$ & $49(53,26 \%)$ & $33(41,25 \%)$ & & $43(64,18 \%)$ & $22(33,84 \%)$ & \\
Bueno (PEIMCP 50-65\%) & $24(26,08 \%)$ & $25(31,25 \%)$ & $p=0,281$ & $15(22,39 \%)$ & $16(24,62 \%)$ & $p=0,000$ \\
Fracaso (PEIMCP $<50 \%)$ & $19(20,65 \%)$ & $22(27,5 \%)$ & & $9(13,43 \%)$ & $27(41,53 \%)$ & \\
\hline
\end{tabular}

Un paciente sometido a BPG, presentó fuga anastomótica que se trató mediante colocación endoscópica de un stent.

5 pacientes tuvieron que ser reintervenidos, 4 del grupo sometido a BPG y uno en el grupo de GV (2 por hemoperitoneo, 2 por perforación intestinal y 1 por absceso intraabdominal).

\section{Éxito de la cirugía bariátrica}

En cuanto al éxito de la cirugía bariátrica a corto plazo, 82 pacientes $(47,7 \%)$ presentan un resultado excelente, 49 pacientes $(28,5 \%)$ bueno y en 41 pacientes $(23,8 \%)$ fracaso de la cirugía bariátrica. A medio plazo, 65 pacientes presentan resultados excelentes $(49,24 \%), 31$ bueno $(23,48 \%)$ y 36 fracaso $(27,27 \%)$. En la Tabla 3 se muestran los resultados según la técnica quirúrgica empleada.

El análisis estadístico, comparando ambas técnicas quirúrgicas, no muestra diferencia en los resultados obtenidos a corto plazo, sin embargo, a medio plazo, existe una diferencia estadísticamente significativa en favor del BPG, presentando mejores resultados.

\section{Pérdida de peso}

En la Figura 1 se muestran las curvas en cuanto a PIMCP y en la Figura 2 las curvas de PEIMCP al año, 2 años, 3 años y 4 años de la cirugía. Se observan diferencias estadísticamente significativas tanto en el PIMCP y PEIMCP entre grupos y a lo largo de los 4 años, exceptuando la PEIMCP al año $(\mathrm{p}=0,1476)$.

\section{Tasa de reganancia de peso a corto y medio plazo}

Se observa una tasa de reganancia de peso a corto plazo del 5,4\%, mientras que a medio plazo alcanza el $14,5 \%$ de los pacientes.

Si comparamos según técnica quirúrgica, se observa una mayor reganancia de peso en el grupo sometido a GV (8 pacientes, 10\%) que en el grupo BPG (1 paciente, $1,09 \%$ ), siendo esta diferencia estadísticamente significativa $(\mathrm{p}=0,009)$.

A medio plazo, se observa reganancia de peso en 6 pacientes sometidos a BPG $(6,66 \%$ de los BPG) y en 19 de las GV (23,75\%), alcanzando la significación estadística $(\mathrm{p}=0,001)$.
Figura 1. Evolución del porcentaje de IMC perdido con ambas técnicas quirúrgicas al año, 2 años, 3 años y 4 años de la cirugía bariátrica mediante by-pass gástrico (BPG) y gastrectomía tubular (GT)

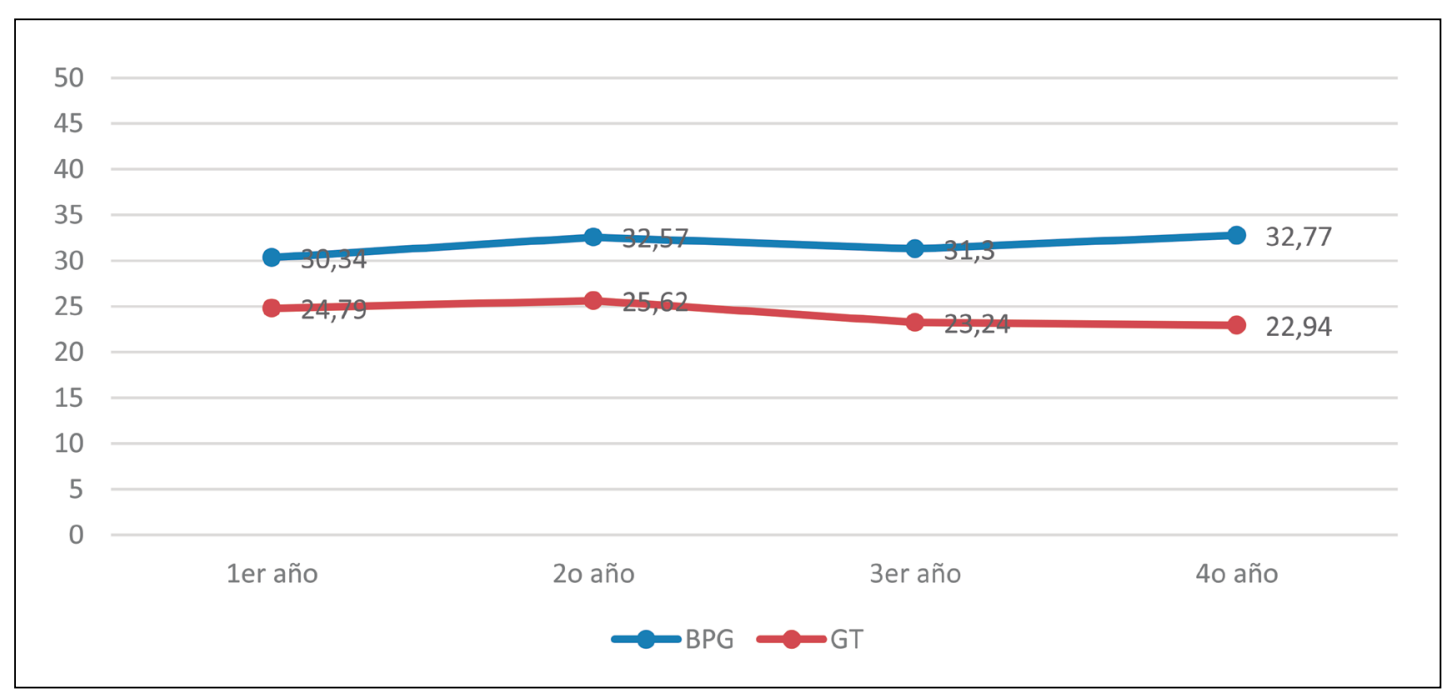




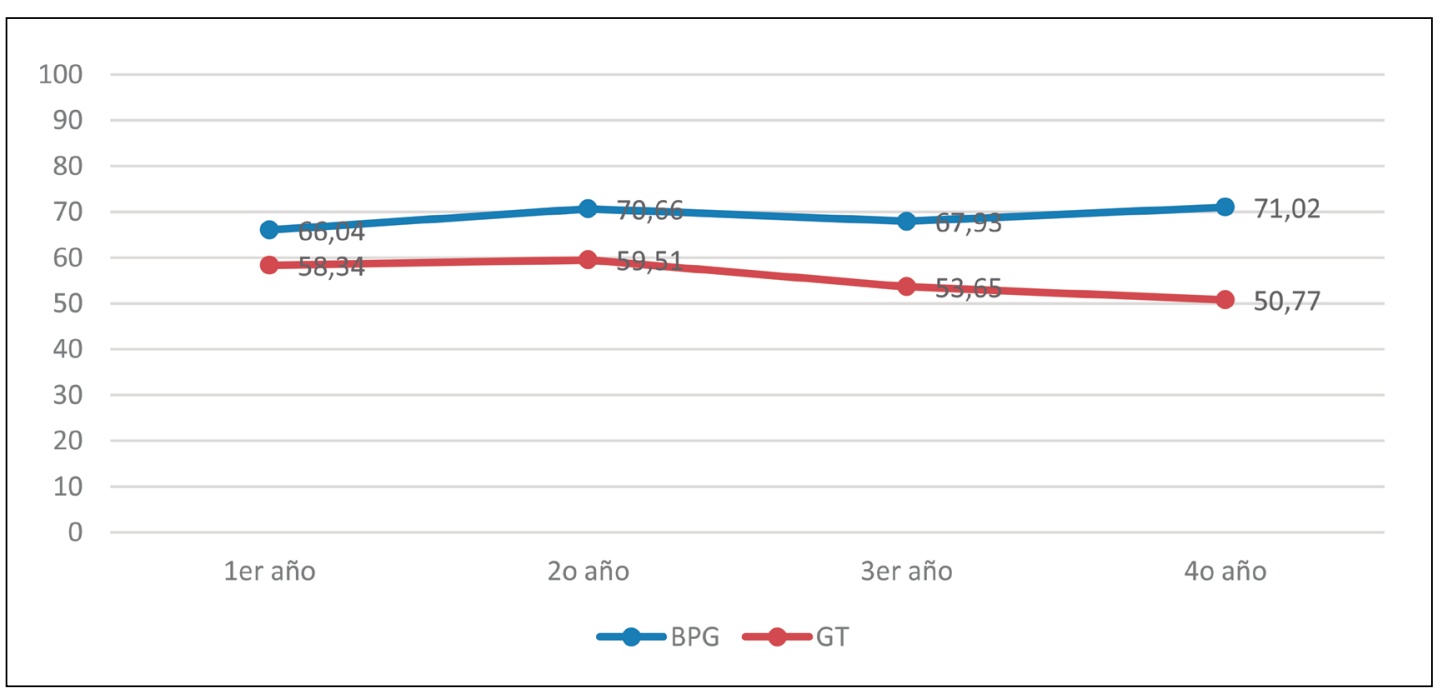

Figura 2. Evolución del porcentaje del exceso de IMC perdido con ambas técnicas quirúrgicas al año, 2 años, 3 años y 4 años de la cirugía bariátrica mediante by-pass gástrico (BPG) y gastrectomía tubular (GT).

\section{Mejoría o resolución de comorbilidades}

En el global de la serie y a medio plazo, se observa una mejoría o resolución de la HTA en el 74,19\% de los pacientes, del $45,76 \%$ en el caso de la DM tipo II y del 51,02\% en el SAOS. En la Figura 3 se muestra el impacto en las comorbilidades asociadas a medio plazo según la técnica quirúrgica.

\section{Discusión}

Como se puede observar en este estudio, la cirugía bariátrica presenta buenos resultados en cuanto a pérdida de peso y a mejoría o resolución de comorbilidades asociadas a la obesidad.

En cuanto al éxito o fracaso de la cirugía bariátrica, se observan resultados excelentes o buenos a corto plazo en aproximadamente el $75 \%$ de los casos (79,34\% de los BPG y $72,5 \%$ de las GV), mientras que a medio plazo se observa un leve descenso en el éxito de ambos procedimientos, alcanzando el $72,5 \%$ de éstos, además de observarse una distribución distinta respecto a los resultados a corto plazo (el BPG presenta mejores resultados, con una tasa de éxito en el $86,6 \%$ de los procedimientos versus un $58,46 \%$ de las GV). Estos resultados podrían explicarse por la mayor reganancia de peso que experimentan los pacientes sometidos a GV. Diversos mecanismos acerca de la reganancia de peso en pacientes sometidos a GV han sido descritos, entre ellos la realización de un reservorio gástrico $>225$ $\mathrm{ml}^{12}$, la dilatación del mismo ${ }^{13} \mathrm{y}$ una dieta de mala calidad y excesivamente calórica. En esta serie, en todos los pacientes sometidos a GV se ha realizado

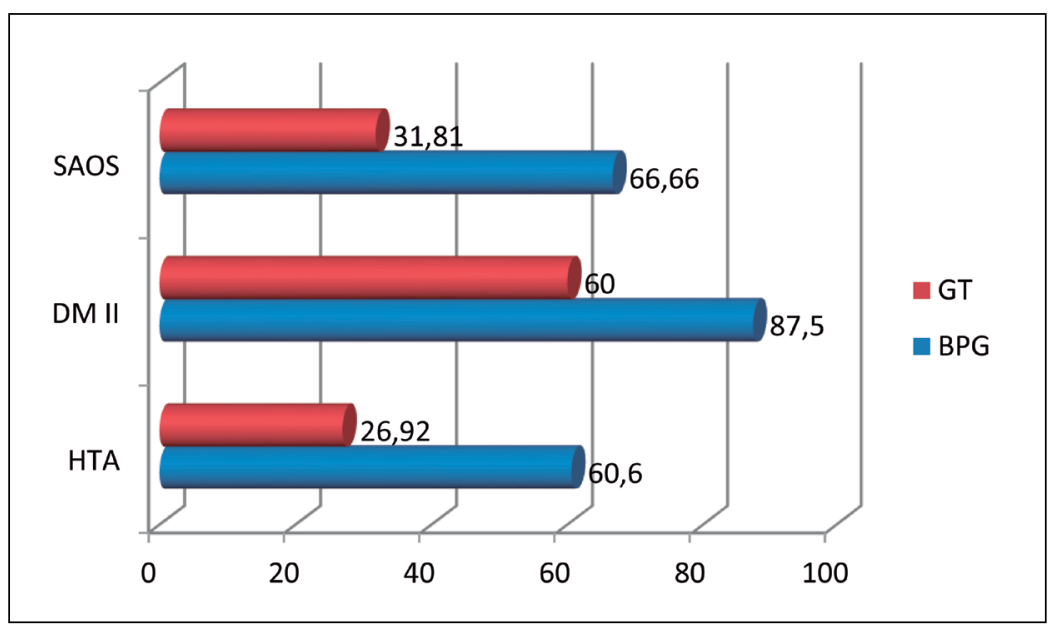

Figura 3. Mejoría o resolución de las comorbilidades con la cirugía bariátrica medio plazo según la técnica quirúrgica empleada. HTA: hipertensión arterial; DM II: diabetes mellitus II; SAOS: síndrome de apnea del sueño; GT: gastrectomía tubular; BPG: Bypass gástrico.

un reservorio gástrico de las mismas características (sobre sonda tutor de 36 French) y todos han recibido el mismo seguimiento y pautas dietéticas.

A pesar de presentar una mayor tasa de complicaciones el grupo sometido a BPG, probablemente relacionada con la mayor complejidad de la técnica, ésta no alcanza la significación estadística. Existe una baja morbilidad asociada a la cirugía bariátrica, con una tasa de complicaciones menores del 12,79\% y mayores del $4,65 \%$ ( 8 pacientes) de los cuales 5 requirieron reintervención.

En cuanto a los resultados acerca de la pérdida de peso, se observan mejores resultados tanto en el PIMCP y PEIMCP en los pacientes sometidos a BPG, 
siendo esta diferencia más notoria con el paso del tiempo tras la intervención quirúrgica, probablemente debido a la reganancia de peso que experimentan los pacientes sometidos a GV.

En este estudio, se observan resultados satisfactorios a corto y medio plazo en cuanto a mejoría de comorbilidades en el $74,19 \%$ de los pacientes que padecen HTA, en el $45,76 \%$ de los pacientes con DM-II y en el $51,02 \%$ de los pacientes con SAOS, siendo estos resultados superiores en el grupo sometido a BPG. Existe poca literatura que compare los resultados obtenidos con el BPG y la GV a medio y largo plazo, sí existiendo datos a corto plazo que muestran resultados similares con ambas técnicas ${ }^{14-15}$.

En esta serie, los resultados obtenidos en cuanto a pérdida de peso, reganancia y mejoría de las comorbilidades, son superiores con el BPG. Estos resultados y aun observando una mayor tasa de complicaciones en el grupo sometido a BPG, nos han llevado a realizar un menor número de $\mathrm{GV}$ en favor del BPG. En 2011, el 47,7\% de las cirugías bariátricas realizadas eran BPG mientras que un
$52,2 \%$ eran GV. En 2015, el porcentaje de BPG alcanzaba el $81 \%$.

Limitaciones de este estudio son el carácter retrospectivo y el sesgo de selección de pacientes según los criterios de indicación quirúrgica mencionados. Otra de las limitaciones es el seguimiento a corto y medio plazo, sin poder obtener resultados a largo plazo.

En conclusión, el BPG ofrece mejores resultados que la GV en pérdida ponderal y en el control de comorbilidades asociadas a corto y medio plazo.

\section{Responsabilidades éticas}

Protección de personas y animales. Los autores declaran que para esta investigación no se han realizado experimentos en seres humanos ni en animales.

Confidencialidad de los datos. Los autores declaran que en este artículo no aparecen datos de pacientes.

Derecho a la privacidad y consentimiento informado. Los autores declaran que en este artículo no aparecen datos de pacientes.

\section{Referencias}

1. Fleming $\mathrm{T}$, Robinson $\mathrm{M}$, Thomson B, Graetz N, Margono C, Mullany EC, et al. Global, regional, and national prevalence of overweight and obesity in children and adults during 1980-2013: a systematic analysis for the Global Burden of Disease Study 2013. Lancet 2014;384:766-81.

2. Swinburn BA, Sacks G, Hall KD, McPherson K, Finegood DT, Moodie ML, et al. The global obesity pandemic: shaped by global drivers and local environments. Lancet 2011;378(9793):804.

3. Sjöström L. Review of the key results from the Swedish Obese Subjects (SOS) trial - a prospective controlled intervention study of bariatric surgery. J Intern Med. 2013;273:219.

4. Angrisani L, Santonicola A, Iovino P, Formisano G, Buchwald H, Scopinaro N. Bariatric Surgery Worldwide 2013. Obes Surg. 2015;25:1822-32.

5. Lakdawala MA, Bhasker A, Mulchandani D, Goel S, Jain S. Comparison between the results of laparoscopic sleeve gastrectomy and laparoscopic Roux-en-Y gastric bypass in the Indian population: a retrospective 1 year study. Obes Surg. 2010;20:1-6.
6. Vidal P, Ramón JM, Goday A, Benaiges D, Trillo L, Parri A, et al. Laparoscopic gastric bypass versus laparoscopic sleeve gastrectomy as a definitive surgical procedure for morbid obesity. Mid-term results. Obes Surg. 2013;23:292-9.

7. Lauti M, Kularatnal M, Hill A.G, MacCormick A.D. Weight Regain Following Sleeve Gastrectomy- a Systematic Review. Obes Surg. 2016;26:1326-34.

8. Cooper TC, Simmons EB, Webb K, Burns JL, Kushner RF. Trends in Weight Regain Following Roux-en-Y Gastric Bypass (RYGB) Bariatric Surgery. Obes Surg. 2015;25:1474-81.

9. Larrad A, Sánchez-Cabezudo C. Indicadores de calidad en cirugía bariátrica y criterios de éxito a largo plazo. Quality indicators in bariatric surgery and criteria for long-term success. Cir Esp. 2004;75:301-4.

10. Brethauer SA, Kim J, el Chaar M, Papasavas P, Eisenberg D, Rogers A, et al. Standarized outcomes reporting in metabolic and bariatric surgery. Surg Obes Relat Dis. 2015;11:489-506.

11. Deguines JB, Verhaeghe P, Yzet T, Robert B, Cosse C, Regimbeau JM. Is the residual gastric volume after laparoscopic sleeve gastrectomy an objective criterion for adapting the treatment strategy after failure? Surg Obes Relat. 13;9:660-6.

12. Braghetto I, Cortes C, Herquiñigo D, Csendes P, Rojas A, Mushle M, et al. Evaluation of radiological gastric capacity and evolution of the BMI 2-3 years after sleeve gastrectomy. Obes Surg. 2009;19:1262-9.

13. Helmiö M, Victorzon M, Ovaska J, Leivonen M, Juuti A, Jaser N, et al. SLEEVEPASS: A randomized prospective multicenter study comparing laparoscopic sleeve gastrectomy and gastric bypass in the treatment of morbid obesity: preliminary results. Surg Endosc. 2012;26:2521-6.

14. Chouillard EK, Karaa A, Elkhoury M, Greco VJ. Laparoscopic Roux-en-Y gastric bypass versus laparoscopic sleeve gastrectomy for morbid obesity: case control study. Surgery for Obesity and Related Diseases 2011;7:500-5.

15. Lakdawala MA, Bhasker A, Mulchandani D, Goel S, Jain S. Comparison between the results of Laparoscopic Sleeve Gastrectomy and Laparoscopic Roux-en-Y Gastric Bypass in the Indian Population: A Retrospective 1 Year Study. Obes Surg. 2010;20:1-6. 